Review

\title{
Keeping an Eye on Myocilin: A Complex Molecule Associated with Primary Open-Angle Glaucoma Susceptibility
}

Farid Menaa ${ }^{1, *}$, Carolina Ayumi Braghini ${ }^{1}$, Jose Paulo Cabral De Vasconcellos ${ }^{2}$, Bouzid Menaa ${ }^{3}$, Vital Paulino Costa ${ }^{2}$, Eugênio Santana De Figueiredo ${ }^{2}$ and Monica Barbosa De Melo ${ }^{1}$

1 Laboratory of Human Genetics, Center for Molecular Biology and Genetic Engineering (CBMEG), University of Campinas (UNICAMP), Campinas-SP 13083-875, Brazil;

E-Mails: carolina.ayumi@cbmeg.unicamp.br (C.A.B.); melomb@uol.com.br (M.B.D.M.)

2

Department of Ophthalmology, Faculty of Medical Sciences, University of Campinas (UNICAMP), Campinas-SP 13083-888, Brazil; E-Mails: cabraljp@uol.com.br (J.P.C.D.V.);

vp.costa@uol.com.br (V.P.C.); eugsantana@ig.com.br (E.S.D.F.)

3 Department of Chemistry and Nanobiotechnology, Fluorotronics, Inc., San Diego, CA 92081, USA; E-Mail: bouzid.menaa@gmail.com (B.M.)

* Author to whom correspondence should be addressed; E-Mails: fmenaa@cbmeg.unicamp.br; or dr.fmenaa@gmail.com; Tel.: +55-19-3521-1138; Fax:+55-19-3521-1089.

Received: 16 June 2011 / Accepted: 21 June 2011 / Published: 27 June 2011

Abstract: $M Y O C$ encodes a secretary glycoprotein of 504 amino acids named myocilin. MYOC is the first gene to be linked to juvenile open-angle glaucoma (JOAG) and some forms of adult-onset primary open-angle glaucoma (POAG). The gene was identified as an up-regulated molecule in cultured trabecular meshwork (TM) cells after treatment with dexamethasone and was originally referred to as trabecular meshwork-inducible glucocorticoid response (TIGR). Elevated intraocular pressure (IOP), due to decreased aqueous outflow, is the strongest known risk factor for POAG. Increasing evidence showed that the modulation of the wild-type (wt) myocilin protein expression is not causative of glaucoma while some misfolded and self-assembly aggregates of mutated myocilin may be associated with POAG in related or unrelated populations. The etiology of the disease remains unclear. Consequently, a better understanding of the molecular mechanisms underlyingPOAG is required to obtain early diagnosis, avoid potential disease progression, and develop new therapeutic strategies. In the present study, we review and discuss the most relevant studies regarding structural characterizations, expressions, molecular interactions, putative functions of MYOC gene and/or its corresponding protein in POAG etiology. 
Keywords: glaucoma; blindness; myocilin; gene variants; protein folding; molecular aggregation

\section{Introduction}

\subsection{Physiopathology of POAG}

Glaucoma includes a group of eye disorders characterized by progressive cupping of the optic nerve head and visual field loss [1]. In fact, this optic neuropathy includes the (i) loss of neural tissue; (ii) activation of glial cells; (iii) tissue remodeling and change of intra-ocular pressure (IOP) that may lead to apoptosis of the retinal ganglion cells [1,2]. One of the mechanisms involved in apoptosis of the retinal ganglion cells includes posterior bowing of the lamina cribrosa, followed by the blockage of the axonal transport and interruption of the delivery of neurotrophins from the superior colliculus to the retinal ganglion cell body [3]. Untreated glaucoma is the leading cause of irreversible blindness in the world. A model projection revealed that, by 2020, 79.6 million of people worldwide will be affected by glaucoma and 5.9 million of individuals will be concerned by glaucoma-associated bilateral blindness [4].

Primary open-angle glaucoma (POAG), also named chronic glaucoma, is the most common form of glaucoma [4]. Unlike angle-closure glaucoma (ACG), aka acute or narrow-angle glaucoma, OAG has a wide and open angle between the iris and cornea (Figure 1). There are several risk factors associated with POAG, including demographic, familial, systemic and ocular factors [5]. Both increased age and ancestry are risk factors indentified in transversal and longitudinal studies. The presence of a family history of glaucoma, another well-established risk factor for the development of POAG, has been described among populations of different ethnicities [5]. The early-onset form of POAG, called juvenile open-angle glaucoma (JOAG), frequently shows a Mendelian pattern of inheritance, whereas the most prevalent sub-form, called adult-onset POAG, is inherited as a complex trait in the majority of the cases [6]. Systemic factors, including low perfusion pressure of the optic nerve head, have also been reported in various studies [7]. Finally, increased IOP is a strong risk factor for POAG. Population-based data have shown a positive association between IOP and the incidence of glaucoma [5].

Figure 1. Comparative retinography photograph from the same patient. The optic disc is showed as a bright area where blood vessels converge.

A. POAG eye (right eye)

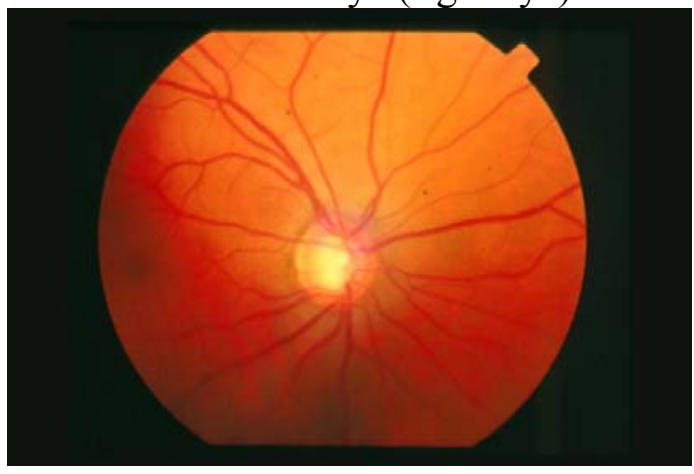

B. Normal eye (left eye)

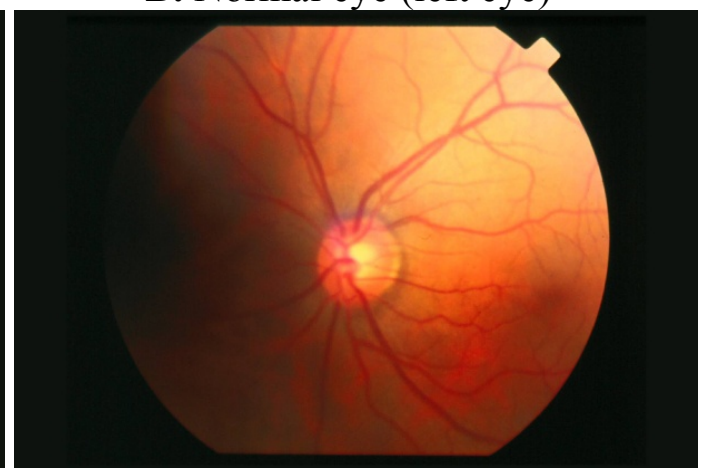


IOP is essential to keep the shape of the eye, allowing the images to be focused accurately onto the retina, and to maintain an adequate intraocular metabolism. The aqueous humor, produced in the ciliary body, contains oxygen and nutrients to nourish the asnterior segment of the eye. It is drained from the eye into the bloodstream through the sieve-like trabecular meshwork (TM). TM is thought to regulate aqueous humor outflow to control IOP. In brief, TM is composed of cells and matrix and is represented by three structurally different regions: (i) the inner uveal meshwork; (ii) the deeper corneoscleral meshwork; (iii) the juxtacanalicular tissue (aka the cribriform meshwork), which is directly adjacent to the inner wall of Schlemm's canal [8]. In POAG eyes, there is a partial blockage within the TM, especially at the juxtacanalicular tissue, restricting the drainage of aqueous humor and increasing IOP (Figure 2). There is also an increase of the extracellular matrix, and an accumulation of banded fibrillar elements that are embedded in different glycoproteins, denominated "plaque material", leading to an increase of the trabecular outflow resistance [9].

Figure 2. Partial side view of the human eye. Red arrows show the drainage of the aqueous humor from the inner chamber to the Schlemm's canal at the outer edge of the iris. In an open angle glaucoma (OAG) eye, fluid is unable to exit at the angle and stays within the eye, thus increasing the pressure (IOP).

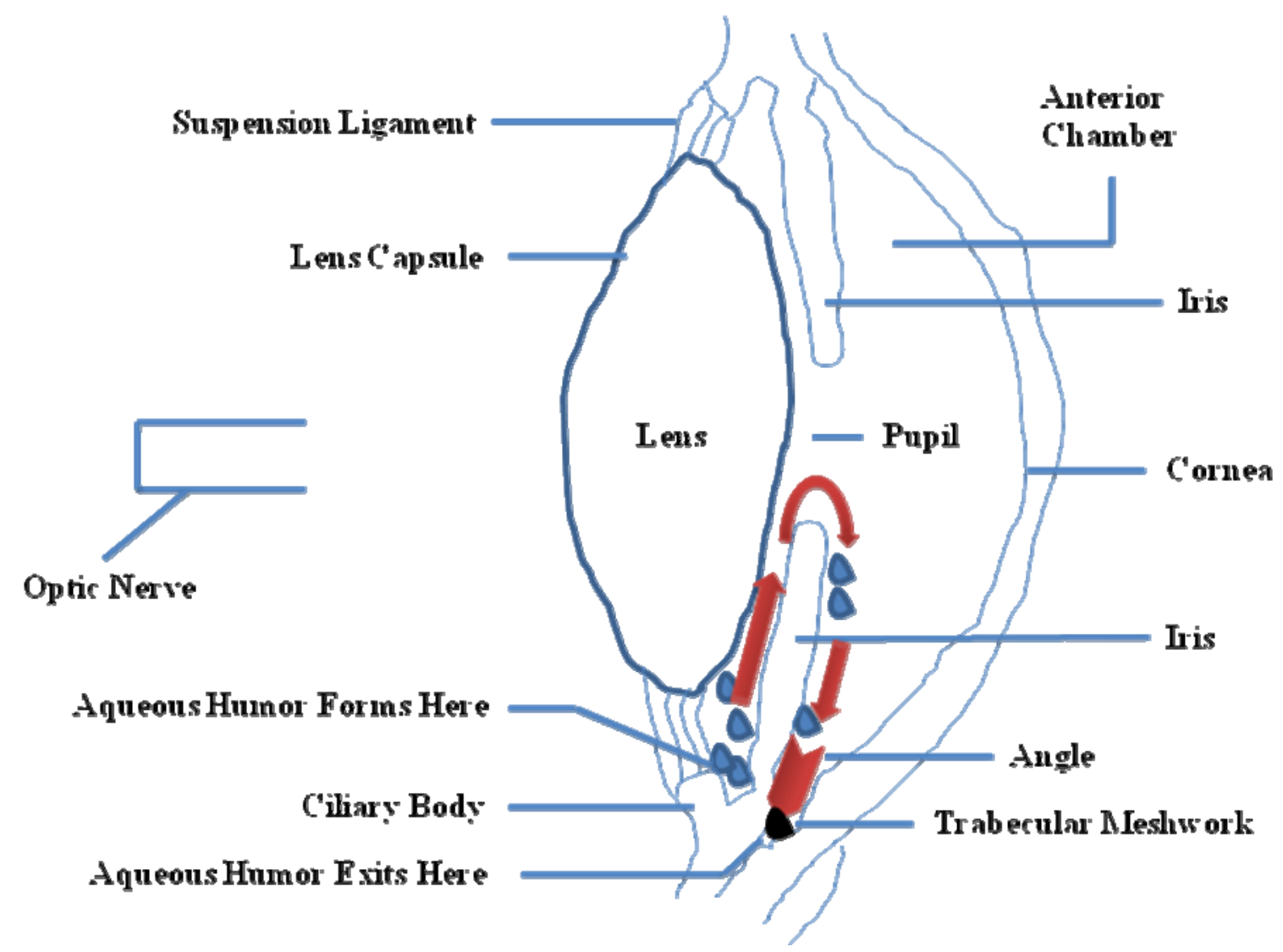

It has been difficult to find a single marker protein to identify a human TM cell, although the pronounced phagocytosis rate shown by these cells can be used as a specific behavior [10]. One of the most important characteristic of the human TM cell is the increased expression of genes such MYOC following exposure to glucocorticoids (e.g., dexamethasone) [10-12], or $\alpha B$ Crystallin, after transforming growth factor- $\beta$ (TGF- $\beta$ ), heat shock or oxidative stress treatment [10]. 
There are usually no symptoms in the initial stages of the glaucomatous disease, but blindness can be prevented if glaucoma is diagnosed and treated early enough. The aim of treatment is to lower the target IOP. Target IOP can be defined as the IOP level where further damage to the optic nerve is likely to be prevented or delayed. Target IOP varies from case to case and depends on age, baseline, untreated IOP, and glaucoma severity [13]. IOP can be lowered by reducing aqueous humor production or by increasing aqueous humor outflow [14]. Indeed, IOP lowering is possible with the use of eye drops, laser treatment directed to TM, which causes ciliary body destruction or, trabeculectomy, a surgical procedure that includes the creation of a guarded fistula to allow communication between the anterior chamber and the subconjunctival space, reducing the resistance to aqueous humor outflow.

Eventually, as suggested from epidemiological and molecular studies, it is now well established that a genetic component may contribute to $\mathrm{OAG}$, and several OAG-associated genes have been identified. The first-identified and the most-studied gene is MYOC, encoding for the myocilin protein, which is highly expressed in and secreted by the human TM [12]. Dominant mutations in MYOC are more frequently observed in JOAG (10\% to $30 \%$ of the cases) than in POAG (3\% to $4 \%$ of patients) $[15,16]$. Some mutations in the MYOC gene lead to the inhibition of mutated myocilin secretion. Secretion of wild-type myocilin (wt myoc) can also be reduced or blocked in the presence of mutated myocilin (mt myoc) [17-19]. It has been suggested that the intracellular accumulation of myocilin aggregates is deleterious to the TM cells, resulting in the deterioration of their function and subsequent elevation of IOP [20,21]. In the present study, we review and discuss relevant literature data about the human myocilin molecule with regards to its structure, expression, interactions and potential role(s) in POAG etiology.

\subsection{Gene Mapping of MYOC}

In 1993, Sheffield et al. performed a linkage analysis in a five generation family with JOAG using microsatellites as genetic markers. The authors described the first locus associated with OAG, denominated GLC1A, mapped in the long arm of chromosome 1 [22]. In 1997, from families affected by autosomal dominant JOAG and POAG, Stone et al. identified mutations in the MYOC gene, a TM-inducible glucocorticoid response gene (TIGR), located in the GLC1A interval on chromosome 1q23-q24 [12]. The cDNA was then independently cloned from subtracted ciliary body [23] and retinal cDNA libraries [24].

\subsection{Expression and Regulation of MYOC}

The induction of the MYOC gene expression was initially observed in cultured TM cells following treatment with glucocorticoids such as dexamethasone (DEX) [11,25]. It is well known that the long-term use of topical ophthalmic steroids results in IOP with glaucoma, known as steroid-induced glaucoma (SIG) [26]. Interestingly, the profile of MYOC up-regulation by DEX was dose- and time-dependent, very similar to the course of development of SIG [25]. Nevertheless, the association between myocilin expression and steroid-induced IOP was not evident [27].

Although MYOC gene has been found, by Northern blot, to be expressed as a $2.3 \mathrm{~kb}$ transcript in many human tissues (e.g., heart, stomach, thyroid, bone marrow, thymus, prostate, colon) but not in all (e.g., brain, placenta, liver, kidney, spleen, or leukocytes), its highest abundance appeared to be restricted to ocular tissues such as iris, ciliary body, optic nerve, aqueous humor and TM [24,28-34]. 
Another study revealed, also by northern blot analysis, that MYOC is variably expressed as 2.1 and $1.8 \mathrm{~kb}$ transcript isoforms in eye structures [35].

The study of regulatory mechanisms governing glucocorticoid-mediated MYOC induction in human TM cells, showed that (i) the promoter region between -2548 and $-1541 \mathrm{bp}$, is required for DEX induction of MYOC expression; (ii) MYOC is a delayed secondary glucocorticoid-responsive gene; (iii) MYOC mRNA is intrinsically quite stable [36]. This MYOC mRNA stability can be regulated by over-expression of optineurin, another protein associated with glaucoma [37].

\subsection{Structural Characterization of MYOC}

Genomic sequence analysis revealed that MYOC gene is composed of three exons of 604,126 , and $782 \mathrm{bp}$, respectively [38], which spans $16 \mathrm{~kb}$ [39]. Independently, another study confirmed the presence of these exons, and an imperfect palindromic glucocorticoid response element in the 5-prime untranslated region (5'-UTR) was identified [40]. In fact, the 5'-UTR of MYOC gene contains TATA and CAT boxes, MIR and Alu repeat sequences, binding sites for multiple hormone and cell signaling response elements, but it lacks SP1-binding sites [25]. The 3'-UTR contains three polyadenylation signal sequences [35] and not two as previously described in [25]. The encoded protein, myocilin, belongs to a family of glycosylated proteins containing a C-terminal olfactomedin-like (OLF) domain [41-43]. This domain was originally identified in a glycoprotein isolated from the olfactory epithelium of frogs [44]. This family includes both secreted and membrane-bound proteins with a characteristic distribution in different tissues [45-53].

Sequence analysis revealed that human MYOC encodes a secreted glycoprotein, myocilin, of a molecular weight of about $55 \mathrm{kDa}$ represented by 504 amino acids, which displays a leucine zipper domain, 10 putative phosphorylation sites and four potential glycosylation sites [38]. Nevertheless, a variant of MYOC with a $338 \mathrm{bp}$ internal deletion removing the coding sequence for the entire leucine zipper region has also been identified in human ocular tissues [35]. Furthermore, it has been observed in aqueous humor and ocular tissues that the human wtmyoc was proteolytically cleaved between arg226 and ile227, resulting in a $35 \mathrm{kDa}$ fragment containing the C-terminal OLF domain and a $20 \mathrm{kDa}$ fragment containing the N-terminal leucine zipper domain [54].

Interestingly, a knowledge-based consensus modeling approach [55,56] showed that myocilin is structurally characterized of three main regions (Figure 3): (i) a N-terminal myosin-like coiled-coil region including a leucine-zipper (between amino-acids 111 and 184); (ii) a flexible linker region (between amino-acids 185 and 245); (iii) a C-terminal OLF domain (between amino-acids 246 and 504). However, this described model is somehow in partial discordance with a deletion experiment

study [57] revealing, a (i) coiled-coil domain located between the amino-acids 78-105; (ii) leucine zipper region between the amino-acids 114-183; (iii) C-terminal domain between the amino-acids 245-504. Functional analysis of myocilin showed that the integrity of amino-terminal coiled-coil regions and olfactomedin homology domain are essential for extracellular adhesion and secretion, the N-terminal region being also important for extracellular interactions (ECM and/or cell surface) [57].

Up-to-date, date the 3D structure of the myocilin is unknown. Indeed, cellular studies have demonstrated temperature-sensitive secretion of myocilin mutants, but difficulties in expression and 
purification have precluded biophysical characterization of wt myoc and disease-causing mutants in vitro [58].

Figure 3. Structure of human myocilin. Colored areas mark the position of the signal peptide, the leucine zipper-like motif and myosin-like domain ( $\mathrm{N}$-terminal region), as well as the olfactomedin (OLF)-like domain (C-terminal region). Adapted from [55].

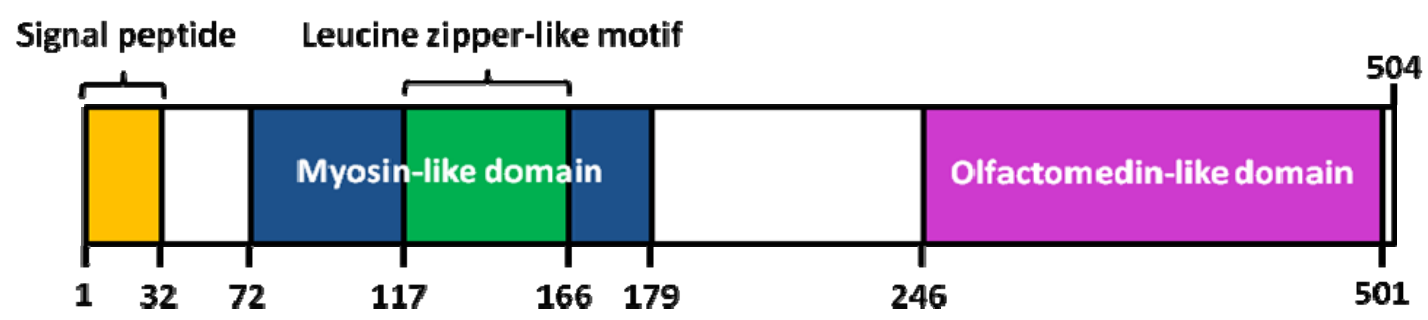

\subsection{Functions of MYOC}

Although the MYOC gene has been studied for more than 10 years, the role of myocilin in the POAG etiology is still poorly understood $[55,59]$. It has been initially speculated that myocilin may cause increased IOP by reduction of the aqueous outflow [12]. Its expression in TM and ciliary body structures involved in the IOP regulation was consistent with this hypothesis [35,37].

Nevertheless, mice with targeted disruption of the MYOC gene $\left(\mathrm{Myoc}^{-/}\right.$) were: (i) viable; (ii) fertile; (iii) without any discernible phenotype; (iv) with a normal IOP, indicating that POAG is not caused by MYOC haplo-insufficiency but, might be due to a gain of function [60]. Furthermore, the over-expression of wt myoc to a level similar to that induced by glucocorticoids in the eyes of transgenic mice did not elevate IOP or display any OAG phenotype [61].

Concordantly, a patient examined with a complex deletion of the maternal copy of chromosome 1, including the entire MYOC gene, did not present elevated IOP or signs of OAG [62]. Finally, the absence of OAG phenotype in an elderly woman homozygous for the Arg46Stop mutation [63] along with the absence of glaucoma in people hemizygous for MYOC [62], suggested that the loss of functional myocilin is not critical for glaucoma development or for normal eye functioning.

Interestingly, elevated amounts of wt myoc in the aqueous humor of transgenic mice caused significant changes in expression of genes involved in cell adhesion and cell-matrix interactions, supporting a role for myocilin in modulating cellular adhesion [64].

Eventually, it seems that wt myoc alone does not play a major role in the POAG etiology but rather through certain molecular (DNA-protein or protein-protein) interaction that may regulate IOP (see Section 2.1). Most importantly, a glaucoma phenotype appeared to be dependent upon expression and structural conformation of some myocilin mutants in ocular tissues (see Section 2.2).

\section{Results and Discussion}

\subsection{Myocilin-Molecules Interactions in POAG Etiology}

Biochemical and immunoelectron microscopic studies data indicated that myocilin may interact with itself and/or with several intracellular and extracellular matrix (ECM) proteins (e.g., flotillin-1, 
$\gamma$-synuclein, hevin-1, optimedin, GAPDH, fibronectin, fibrillin-1 or type VI collagen) [52,65-73], but the biological significance and clinical relevance of such interactions in the POAG etiology remain unclear. Thereby, myocilin forms in vivo (e.g., human aqueous humor, human TM) high molecular weight complexes, ranging from 120 to $180 \mathrm{kDa}$, due to interaction with itself and other myocilin binding proteins $[65,74]$.

Among the possible molecular interactions that may modulate IOP and thus prevent or contribute to POAG, we can cite: (i) the myocilin-myocilin one, involving the amino-acids 117-166 in the leucine zipper domain, which might be necessary for the myocilin-RLC (myosin regulatory light chain) interaction, suggesting a role for myocilin in the actomyosin system [71,74]; (ii) the interaction of myocilin with components of the Wnt signaling pathways (e.g., Wnt receptors of the Frizzled (Fzd) family, Wnt antagonists of the secreted Frizzled-related protein (sFRP) family and Wnt inhibitory factor 1 (WIF-1)), which modulates the organization of actin cytoskeleton stimulating the formation of stress fibers, critical for the contractility of the TM and IOP regulation [70]. Interestingly, the absence of a glaucoma phenotype resulting from myocilin null mutation $\left(\mathrm{Myoc}^{-/}\right)$in the eye could also be explained by the compensatory action of Wnt proteins [70]; (iii) the interaction between the myocilin variant (-1000G) and the apolipoprotein (APOE) variant (-491T) previously associated with Alzheimer disease (AD). Indeed, this interaction involving a single nucleotide polymorphism (SNP) in the respective promoter is associated with increasing IOP and limited effectiveness of IOP-lowering treatments in patients with POAG [72].

A number of genes regulated by glucocorticoids in ocular tissues, or those for which the expression is specifically modulated in POAG tissues, might represent potential myocilin-interacting candidates that may contribute to the progression of glaucoma (data not shown). Indeed, in addition to MYOC, other genes were found up-regulated in the DEX-induced human TM [75]. For instance, we can cite those coding the serine protease inhibitors $\alpha 1$-antichymotrypsin and pigment epithelium-derived factor (PEDF), the gene coding the interleukin-8 (IL-8), chemokine involved in cell motility or, the gene coding the carbonic anhydrase XII (CA12), implicated in aqueous humor formation and calcification [74]. Furthermore, comparative analysis of human genome-wide gene expression profiles in POAG TM tissues indicated significant expression changes in genes associated with inflammation (e.g., up-regulation of selectin-E) or anti-oxidation (e.g., down-regulation of paraoxonase 3 and ceruloplasmin) [76].

Eventually, extensive identification and characterization of myocilin-interacting proteins by methods such ChIP-on-chip and/or yeast-two hybrid system may shed light the structure and function of myocilin in the TM and aqueous outflow pathway, thus define the functional protein complex(es) surrounding myocilin protein (wild-type or mutated) implicated in the glaucomatous disease.

\subsection{MYOC Variants in POAG Etiology}

The initial step of POAG pathogenesis may depend upon expression of abnormal mutant myocilin protein, which accumulates into the cell instead of being normally secreted $[61,77,78]$. A functional assay consisting in transfecting mammalian cells with MYOC constructs containing sequence changes seen in glaucoma patients has demonstrated the function of MYOC mutations or polymorphisms in the glaucoma pathogenesis [79]. Indeed, the mutants rendered the expressed protein insoluble in the 
detergent Triton X-100 and would interfere with secretion, dimerization, or interaction of myocilin with ECM components of the TM [79].

Several mutations in MYOC gene are continuously described in families with POAG from different ethnical or geographic origins, and mutants databases can freely be accessed online [80]. From those databases, we could notice that the most encountered types of mutations are missense $(\sim 86 \%)$ or nonsense $(\sim 5.5 \%)$.

MYOC mutations account for about $2 \%-4 \%$ of the POAG patients and, for about $10 \%-30 \%$ of JOAG patients $[15,16]$. The majority of mutations (over 90\%) are concentrated in the evolutionarily conserved C-terminal OLF domain encoded by a single exon [81], while other mutations and SNPs have been identified in the promoter, upstream region of the MYOC gene [82-85]. The frequencies of these mutations are usually higher in family studies than in unrelated ones [86].

In vitro and in vivo studies showed that several OLF domain mutations prevented myocilin secretion in physiological temperature conditions, but when cells were cultured at $30{ }^{\circ} \mathrm{C}$, a process known to facilitate protein folding, some sequestered mutants were released in the extracellular medium [57].

One of the major studies carried out in glaucoma patient populations was performed by screening 1703 patients with POAG from five different populations representing three racial groups [87]. There were 1284 patients from Caucasian populations in Iowa (727), Australia (390) and Canada (167) in addition to 312 African American patients from New York City and 107 Asian patients from Japan. Overall, 61 different MYOC sequence variations were identified, 21 of which were judged to be probable disease-causing mutations. Among the 21 mutations, $16(76 \%)$ were found in only one population. The most common mutation observed, Q368X, was observed in 27 of the $1703(1.6 \%)$ glaucoma pro-bands and, at least once, in all groups except the Japanese one [87].

We have identified in a Brazilian family pedigree, by single-strand conformation polymorphism (SSCP) and sequencing analyses, the C433R mutation in the MYOC gene, which was strictly associated with JOAG/POAG [88]. The penetrance of this mutation was $0 \%$ in persons younger than 10 years (0/4), 40\% in those between 11 and 30 years $(2 / 5), 75 \%$ in those aged from 30 to 40 years $(3 / 4)$, and $100 \%$ in those older than 40 years $(4 / 4)$ [88].

Interestingly, mutations in both MYOC and CYP1B1 genes, the later is the most important gene associated with primary congenital glaucoma, were described in a Canadian family segregating both autosomal dominant adult and juvenile onset-POAG [89]. CYP1B1 encodes a member of the cytochrome P450 superfamily and is co-expressed with MYOC in the iris TM, and ciliary body of the eye [89]. All affected family members carried the MYOC mutation but those who also had the CYP1B1 mutation had juvenile onset-POAG, whereas those with only the MYOC mutation had the adult-onset form [89]. The mean age at onset of disease among carriers of the MYOC mutation alone was 51 years, whereas those of both MYOC and CYP1B1 mutations had an average age of only 27 years [89]. Individuals carrying only a CYP1B1 mutation were not clinically affected, so it has been concluded that in this family, CYP1B1 could act as a modifier of MYOC [89].

\subsection{Misfolding and Aggregation of Myocilin in POAG Etiology}

Mutations in proteins that induce misfolding and proteasomal degradation are common causes of inherited diseases [90]. It has been found that POAG-causing myocilin mutants were misfolded, highly 
aggregation-prone, accumulated in large aggregates in the rough ER of human differentiated primary TM cells [21] and formed typical Russel bodies [91].

In TM cells, P370L mutant myocilin, which causes the most severe glaucoma phenotype, was not secreted under normal culture conditions $\left(37^{\circ} \mathrm{C}\right)$, and prolonged expression resulted in abnormal cell morphology and cell killing [21]. However, culture of TM cells at $30^{\circ} \mathrm{C}$ facilitated myocilin folding, promoted secretion of mutant myocilin, normalized cell morphology and, reversed cell lethality [21]. ER stress-induced apoptosis is a pathway to explain the reduction of TM cells in patients with myocilin-caused glaucoma [91]. Indeed, the presence of myocilin aggregates induced the unfolded protein response proteins $\mathrm{BiP}$ and phosphorylated ER-localized eukaryotic initiation factor-2alpha kinase (PERK) with the subsequent activation of caspases 12 and 3 and expression of C/EBP homologous protein (CHOP)/GADD153, leading to apoptosis [91].

From these observations, myocilin-associated POAG can be considered as an ER storage disease, consisting in a progression of events that involves chronic expression of misfolded and non-secreted myocilin, subsequent TM cell death, TM dysfunction and impediment of aqueous humor outflow leading to elevated IOP $[21,91]$.

In accordance with this observation, several glaucoma-associated MYOC mutations, including the P370L one, inhibited calpain II dependent-endoproteolytic processing of full-length myocilin, normally releasing two fragments of $\sim 20 \mathrm{kDa}$ (N-terminal part) and $\sim 35 \mathrm{kDa}$ (C-terminal part), which resulted in accumulation of insoluble mutant myocilin aggregates in the ER [54]. This cleavage might regulate extracellular and matricellular protein interactions (e.g., myocilin-hevin) [67,92], contributing to the control of IOP [93], notably by decreasing myocilin homo-aggregates [94].

In cell culture, the toxicity of mutant myocilins can be reduced by the addition of certain chemical chaperones (e.g., 4-phenylbutyric acid (4-PBA)) or osmolytes (e.g., trimethylamine $N$-oxide (TMAO)) $[90,95]$, and in vitro, the compromised stability of myocilin mutants can be restored with some of the same compounds [58]. Indeed, treatment with 4-PBA of cells reduced the amount of insoluble myocilin aggregates and restored the secretion of mutant myocilin [96]. The same treatment in cells co-expressing wt and mt myocilin relieved ER stress and significantly reduced the rate of apoptosis [96]. The ways that a chaperone may help trafficked proteins, both wt and mt, meet the ER quality control requirements, include both thermodynamic- and kinetics-based mechanisms [58]. For instance, the chaperone could (i) accelerate the protein folding; (ii) bind and stabilize a fully folded protein; (iii) stabilize the protein for post-translational modification or interaction with a binding partner required for proper trafficking; (iv) all or some combination of the above [58].

In this context, the protein folding, misfolding and aggregation processes represent a continuous and necessary challenge to better understand the protein function in a given pathology. Unfortunately, the main factors influencing those processes, with regard to myocilin, remain poorly understood. In a general way, the protein structure not only depends on the amino-acid sequence but also on its micro-local environment which enhances the protein helicity [97-99]. An efficient in vitro strategy to mimic the native in vivo protein structure and determine the influencing parameters of protein folding and aggregation, can be obtained by protein encapsulation using an established non-destructive modified sol-gel glass method [97,99]. Indeed, optically transparent biomaterials can facilitate the determination of the protein structure as well as the molar protein ellipticity, stability and biological activity using spectroscopic techniques, such as circular dichroism spectroscopy (CDS) [98]. 
Interestingly, the first detailed biophysical characterization of MYOC-OLF domain in solution, to gain insight into its structure and function, has been recently reported [100]. Thereby, it has been shown that MYOC-OLF is stable in the presence of glycosaminoglycans (GAGs), as well as in a wide $\mathrm{pH}$ range in buffers with functional groups reminiscent of such GAGs [100]. CDS analysis of the OLF domain revealed significant $\beta$-sheet and $\beta$-turn secondary structure [100]. At neutral $\mathrm{pH}$, intrinsic tryptophan fluorescence and CDS melts indicated a highly cooperative transition with a melting temperature of $\sim 55^{\circ} \mathrm{C}$. Limited proteolysis combined with mass spectrometry (MS) revealed that the compact core structural of $O L F$ domain consists of residues located from position 238 to 461 [100].

\subsection{Myocilin Is Phylogenetically Well Conserved}

Pearson's sequence format along with the multiple sequence alignment software ClustalW2 2.0.12 accessible online $[101,102]$ were used to compare the full-length of myocilin primary sequence of different species $(n=8)$ (Figure 4). First, very few gaps were noticed in the myocilin sequences, and the most important one was observed at the very beginning of the N-terminal sequence where 14 amino-acids are missing in seven species excepted in human (Figure 4). This short amino-acid sequence corresponds to the peptide signal (Figure 3), suggesting human specificity in relation to the myocilin protein cellular trafficking (data not shown). Second, the length of the myocilin protein sequence varied from 483 (i.e., Canis lupus familiaris) to 504 amino-acids (i.e., Homo sapiens) with an average of 491 amino-acids. Finally, pairwise alignments showed the best score identity between Homo sapiens and Macaca fascicularis ( $96 \%$ ), while the lower score was observed between Mus musculus and Bos taurus ( $78 \%$ ). We found that the overall average score of identity, between the eight myocilin sequences was around $77 \%$, demonstrating that myocilin is phylogenetically well conserved.

To estimate the average distance between the eight myocilin sequences and design a phylogram, we used BLOSUM62 program integrated to the ClustalW2 software.

Figure 4. Primary sequence alignment of myocilin from different mammalian species. Protein sequence conservation appears in blue.

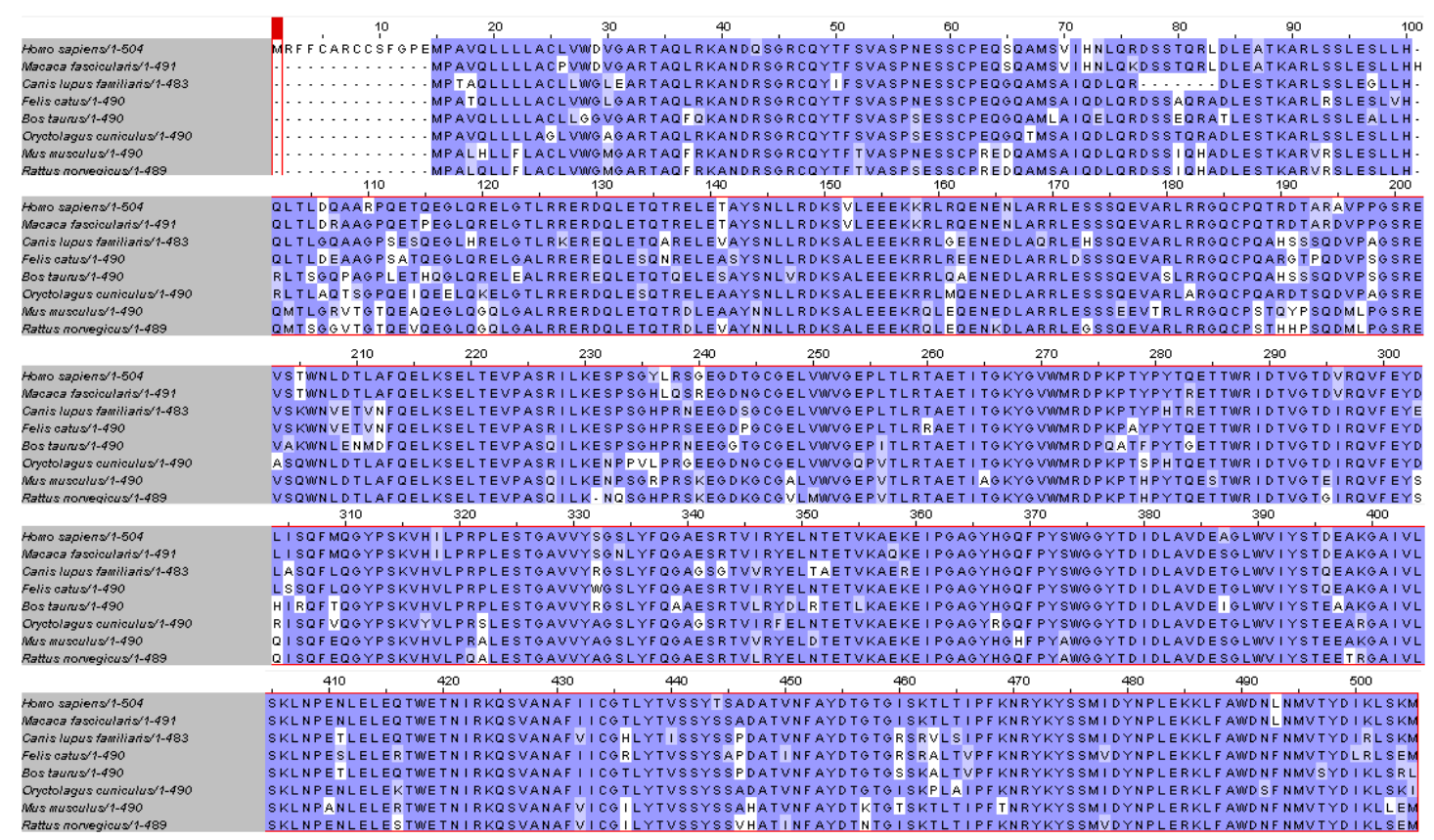


We observed that the most related mammalian species to Homo sapiens are respectively Macaca fascicularis, Mus musculus, Rattus norvegicus, Canis lupus familiaris, Felis catus, Oryctolagus cuiniculus and Bos Taurus (Figure 5).

Figure 5. Phylogenic tree showing average distance between mammalian myocilin primary sequences (arbitrary units).

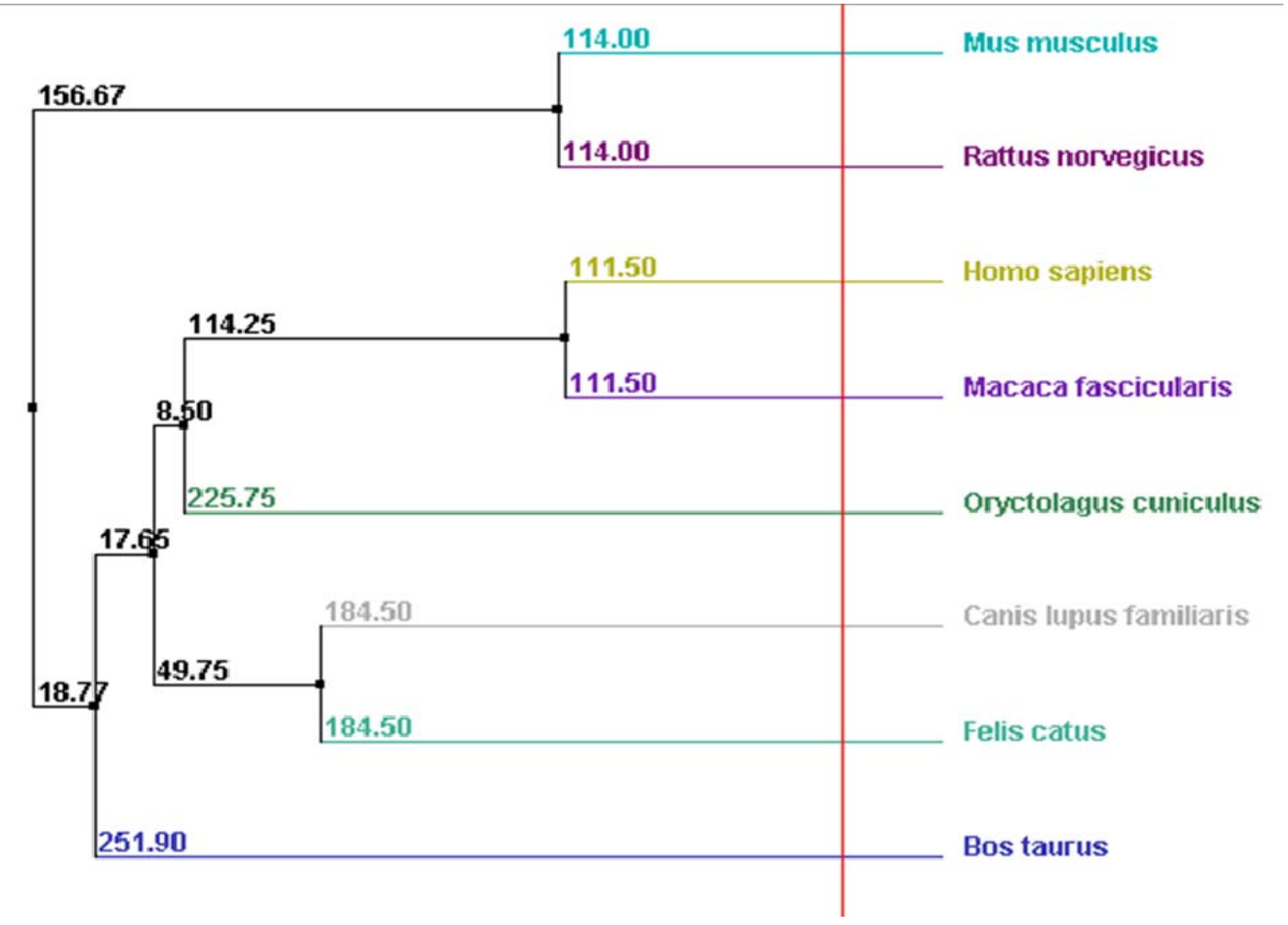

\subsection{Putative 3D-Structure of the Myocilin}

One of the possible approaches to determine myocilin 3D protein structure and predict its functions could be the use of the I-TASSER program [103-105]. Briefly, I-TASSER uses state-of-the-art algorithms and generates full length model of proteins by excising continuous fragments from threading alignments, before reassembling them using replica-exchanged Monte Carlo simulations. 3D models were built based on multiple-threading alignments by LOMETS and iterative TASSER simulations. Function insights were then derived by matching the predicted models with protein function databases such "protein databank (PDB)", available online [106,107]. The most confident predicted protein model in terms of quality/accuracy - based on C-score, TM-score and RMSD of the program - that we found is related to the tRNA wybutosine synthesizing enzyme (TYW4) [108]. TYW4 is an $S$-adenosylmethionine (SAM)-dependent enzyme that catalyzes the final step of wybutosine biosynthesis, methylation and methoxycarbonylation. Based on our preliminary structural studies (Figure 6), we thus suggest that myocilin may be involved in a methylation process. 
Figure 6. Putative 3D-structure of human myocilin.
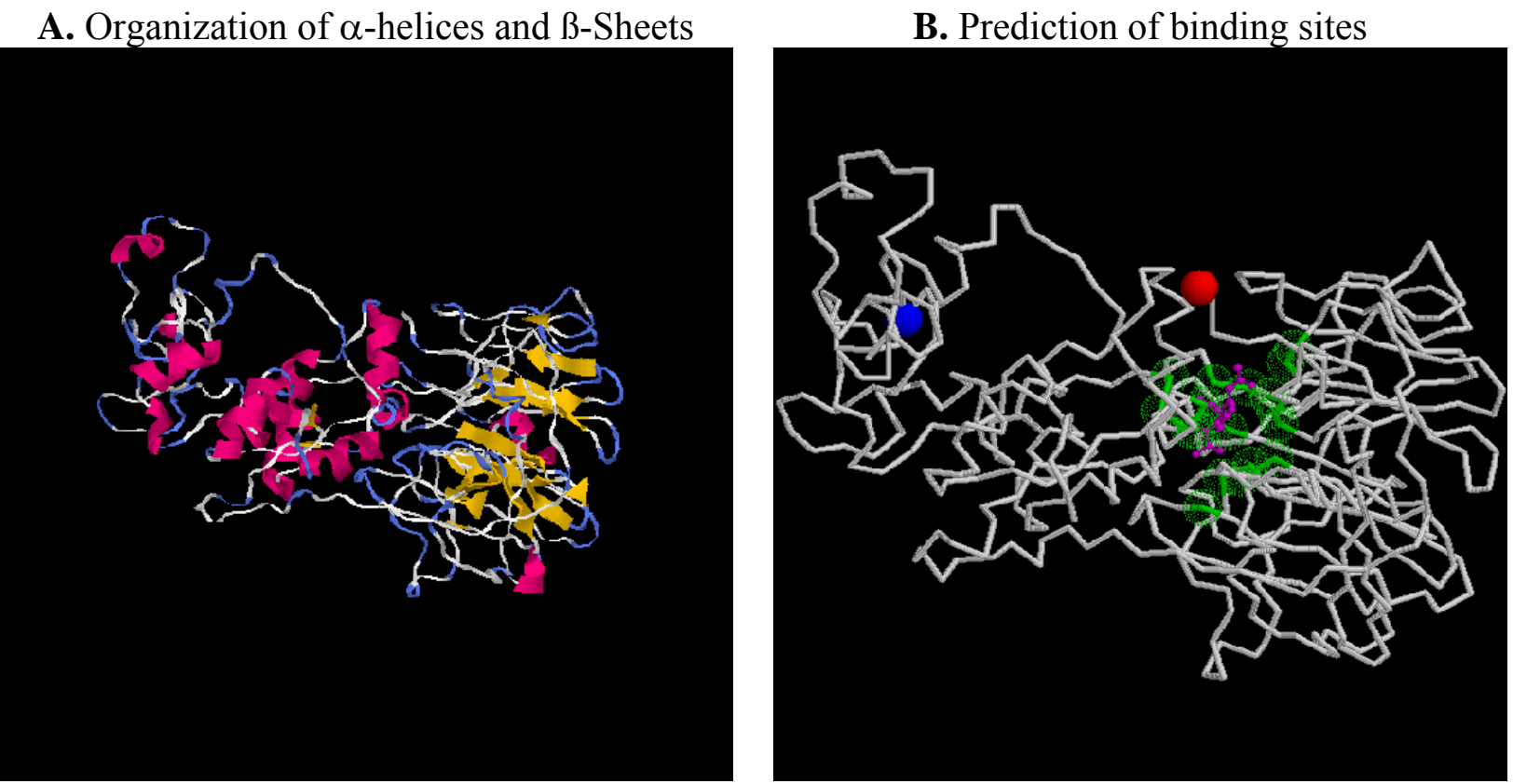

\section{Conclusions}

POAG is an optic neuropathy with high worldwide prevalence and strong evidence of complex inheritance. Although the POAG etiology remains unclear and may involve several genes associated with the disease, it is now relatively well documented that mutations, mostly encountered in OLF domain of the MYOC gene, play a critical role in the manifestation of the disease. One molecular mechanism explaining the possible association of some MYOC mutations and POAG involves the misfolding and aggregation processes of myocilin protein. Indeed, mutated myocilin can accumulate into the ER of TM, form aggregates, suppress the secretion of normal myocilin and blocks the TM in the eye, which eventually cause cell dysfunction and death, increases the IOP, and eventually leads to POAG. Thereby, myocilin P370L mutant would represent, up-to-date, the one that causes the most severe glaucoma phenotype by eliciting the most potent inhibition of the myocilin processing.

Future challenges may include: (i) genome-wide association studies (GWAS) in order to identify new important submicroscopic genomic alterations such as single-nucleotide polymorphisms (SNPs) but also copy number variations (CNVs) (e.g., deletions, duplications) with the final aim to find causative genes involved in glaucoma, and so better understand the etiology of POAG; (ii) further physical and functional characterization of wt myoc versus mutant $\mathrm{mt}$ myoc in different micro-environmental conditions; (iii) characterization of dynamic molecular interactions between myocilin-proteins complexes; (iv) unravelling of the $2 \mathrm{D} / 3 \mathrm{D}$ myocilin structure (wt versus mt myoc); (v) continuous determination of myocilin variants pattern, clinically relevant among worldwide patients populations, in order to establish groups at risk for POAG and allow early diagnosis; (vi) efficient therapy-based control of the IOP-without major post-treatments complications-to avoid potential disease progression. 


\section{Acknowledgements}

The authors gratefully acknowledge Abder Menaa for his critical review of the article as well as FAPESP (Sao Paulo Research Foundation) for its financial support.

\section{References and Note}

1. Flammer, J.; Mozaffarieh, M. What is the present pathogenetic concept of glaucomatous optic neuropathy? Surv. Ophthalmol. 2007, 52, 162-173.

2. Morrison, J.C.; Johnson, E.C.; Cepurna, W.; Jia, L. Understanding mechanisms of pressureinduced optic nerve damage. Prog. Retin. Eye Res. 2005, 24, 217-240.

3. Quigley, H.A. Neuronal death in glaucoma. Prog. Retin. Eye Res. 1999, 18, 39-57.

4. Quigley, H.A.; Broman, A.T. The number of people with glaucoma worldwide in 2010 and 2020. Br. J. Ophthalmol. 2006, 90, 262-267.

5. Leske, M.C. Open-angle glaucoma-An epidemiologic overview. Ophthalmic. Epidemiol. 2007, 14, 166-172.

6. Libby, R.T.; Gould, D.B.; Anderson, M.G.; John, S.W.M. Complex genetics of glaucoma susceptibility. Rev. Genomics Hum. Genet. 2005, 6, 15-44.

7. Leske, M.C. Ocular perfusion pressure and glaucoma: clinical trial and epidemiologic findings. Curr. Opin. Ophthalmol. 2009, 20, 73-78.

8. Tamm, E.R. The trabecular meshwork outflow pathways: Structural and functional aspects. Exp. Eye Res. 2009, 88, 648-655.

9. Tektas, O.Y.; Lütjen-Drecoll, E. Structural changes of the trabecular meshwork in different kinds of glaucoma. Exp. Eye Res. 2009, 88, 769-775.

10. Gasiorowski, J.Z.; Russell, P. Biological properties of trabecular meshwork cells. Exp. Eye Res. 2009, 88, 671-675.

11. Polansky, J.R.; Fauss, D.J.; Chen, P.; Chen, H.; Lütjen-Drecoll, E.; Johnson, D.; Kurtz, R.M.; Ma, Z.D.; Bloom, E.; Nguyen, T.D. Cellular pharmacology and molecular biology of the trabecular meshwork inducible glucocorticoid response gene product. Ophthalmologica 1997, 211, 126-139.

12. Stone, E.M.; Fingert, J.H.; Alward, W.L.M.; Nguyen, T.D.; Polansky, J.R.; Sunden, S.L.F.; Nishimura, D.; Clark, A.F.; Nystuen, A.; Nichols, B.E.; et al. Identification of a gene that causes primary open angle glaucoma. Science 1997, 275, 668-670.

13. Detry-Morel, M. Currents on target intraocular pressure and intraocular pressure fluctuations in glaucoma management. Bull. Soc. Belge Ophtalmol. 2008, 308, 35-43.

14. Clement, C.I.; Goldberg, I. The management of complicated glaucoma. Indian J. Ophthalmol. 2011, 59, 141-147.

15. Fan, B.J.; Wiggs, J.L. Glaucoma: Genes, phenotypes, and new directions for therapy. J. Clin. Invest. 2010, 120, 3064-3072.

16. Shimizu, S.; Lichter, P.R.; Johnson, A.T.; Zhou, Z.; Higashi, M.; Gottfredsdottir, M.; Othman, M.; Moroi, S.E.; Rozsa, F.W.; Schertzer, R.M.; et al. Age-dependent prevalence of mutations at the GLC1A locus in primary open-angle glaucoma. Am. J. Ophthalmol. 2000, 130, 165-177. 
17. Gobeil, S.; Rodrigue, M.A.; Moisan, S.; Nguyen, T.D.; Polansky, J.R.; Morissette, J.; Raymond, V. Intracellular sequestration of hetero-oligomers formed by wild-type and glaucoma-causing myocilin mutants. Investig. Ophthalmol. Vis. Sci. 2004, 45, 3560-3567.

18. Jacobson, N.; Andrews, M.; Shepard, A.R.; Nishimura, D.; Searby, C.; Fingert, J.H.; Hageman, G.; Mullins, R.; Davidson, B.L.; Kwon, Y.H.; et al. Non-secretion of mutant proteins of the glaucoma gene myocilin in cultured trabecular meshwork cells and in aqueous humor. Hum. Mol. Genet. 2001, 10, 117-125.

19. Malyukova, I.; Lee, H.S.; Fariss, R.N.; Tomarev, S.I. Mutated mouse and human myocilins have similar properties and do not block general secretory pathway. Investig. Ophthalmol. Vis. Sci. 2006, 47, 206-212.

20. Joe, M.K.; Sohn, S.; Hur, W.; Moon, Y.; Choi, Y.R.; Kee, C. Accumulation of mutant myocilins in ER leads to ER stress and potential cytotoxicity in human trabecular meshwork cells. Biochem. Biophys. Res. Commun. 2003, 312, 592-600.

21. Liu, Y.; Vollrath, D. Reversal of mutant myocilin non-secretion and cell killing: Implications for glaucoma. Hum. Mol. Genet. 2004, 13, 1193-1204.

22. Sheffield, V.C.; Stone, E.M.; Alward, W.L.; Drack, A.V.; Johnson, A.T.; Streb, L.M.; Nichols, B.E. Genetic linkage of familial open angle glaucoma to chromosome 1q21-q31. Nat. Genet. 1993, 4, 47-50.

23. Ortego, J.; Escribano, J.; Coca-Prados, M. Cloning and characterization of subtracted cDNAs from a human ciliary body library encoding TIGR, a protein involved in juvenile open angle glaucoma with homology to myosin and olfactomedin. FEBS Lett. 1997, 413, 349-353.

24. Kubota, R.; Noda, S.; Wang, Y.; Minoshima, S.; Asakawa, S.; Kudoh, J.; Mashima, Y.; Oguchi, Y.; Shimizu, N.A. novel myosin-like protein (myocilin) expressed in the connecting cilium of the photoreceptor: Molecular cloning, tissue expression, and chromosomal mapping. Genomics 1997, 41, 360-369.

25. Nguyen, T.D.; Chen, P.; Huang, W.D.; Chen, H.; Johnson, D.; Polansky, J.R. Gene structure and properties of TIGR, an olfactomedin-related glycoprotein cloned from glucocorticoid-induced trabecular meshwork cells. J. Biol. Chem. 1998, 273, 6341-6350.

26. Francois, J. Cortisone et tension ocularie. Ann. Ocul. (Paris) 1954, 187, 805-816.

27. Fingert, J.H.; Clark, A.F.; Craig, J.E.; Alward, W.L.; Snibson, G.R.; McLaughlin, M.; Tuttle, L.; Mackey, D.A.; Sheffield, V.C.; Stone, E.M. Evaluation of the myocilin (MYOC) glaucoma gene in monkey and human steroid-induced ocular hypertension. Invest. Ophthalmol. Vis. Sci. 2001, 42, 145-152.

28. Tomarev, S.I.; Wistow, G.; Raymond, V.; Dubois, S.; Malyukova, I. Gene expression profile of the human trabecular meshwork: NEIBank sequence tag analysis. Investig. Ophthalmol. Vis. Sci. 2003, 44, 2588-2596.

29. Karali, A.; Russell, P.; Stefani, F.H.; Tamm, E.R. Localization of myocilin/trabecular meshworkinducible glucocorticoid response protein in the human eye. Investig. Ophthalmol. Vis. Sci. 2000, 41, 729-740. 
30. Wistow, G.; Berstein, S.L.; Ray, S.; Wyatt, M.K.; Behal, A.; Touchman, J.W.; Bouffard, G.; Smith, D.; Peterson, K. Expressed sequence tag analysis of adult human iris for the NEIBank Project: Steroid-response factors and similarities with retinal pigment epithelium. Mol. Vis. 2002, 8, 185-195.

31. Wistow, G.; Berstein, S.L.; Wyatt, M.K.; Fariss, R.N.; Behal, A.; Touchman, J.W.; Bouffard, G.; Smith, D.; Peterson, K. Expressed sequence tag analysis of human RPE/choroid for the NEIBank Project: Over 6000 non-redundant transcripts, novel genes and splice variants. Mol. Vis. 2002, 8, 205-220.

32. Swiderski, R.E.; Ross, J.L.; Fingert, J.H.; Clark, A.F.; Alward, W.L.; Stone, E.M.; Sheffield, V.C. Localization of MYOC transcripts in human eye and optic nerve by in situ hybridization. Investig. Ophthalmol. Vis. Sci. 2000, 41, 3420-3428.

33. Rao, P.V.; Allingham, R.R.; Epstein, D.L. TIGR/myocilin in human aqueous humor. Exp. Eye Res. 2000, 71, 637-641.

34. Russell, P.; Tamm, E.R.; Grehn, F.J.; Picht, G.; Johnson, M. The presence and properties of myocilin in the aqueous humor. Investig. Ophthalmol. Vis. Sci. 2001, 42, 983-986.

35. Kong, T.H. Post-transcriptional modification of the gene genetically linked to juvenile open-angle glaucoma: novel transcripts in human ocular tissues. Gene 2001, 280, 115-122.

36. Joe, M.K.; Sohn, S.; Kim, T.E.; Im, J.E.; Choi, Y.R.; Kee, C. Analysis of glucocorticoid-induced MYOC expression in human trabecular meshwork cells. Vis. Res. 2011, 51, 1033-1038.

37. Park, B.C.; Tibudan, M.; Samaraweera, M.; Shen, X.; Yue, B.Y. Interaction between two glaucoma genes, optineurin and myocilin. Genes Cells 2007, 12, 969-979.

38. Fingert, J.H.; Ying, L.; Swiderski, R.E.; Nystuen, A.M.; Arbour, N.C; Alward, W.L.M.; Sheffield, V.C.; Stone, E.M. Characterization and comparison of the human and mouse GLC1A glaucoma genes. Genome Res. 1998, 8, 377-384.

39. Tomarev, S.I.; Tamm, E.R.; Chang, B. Characterization of the mouse Myoc/Tigr gene. Biochem. Biophys. Res. Commun. 1998, 245, 887-893.

40. Kubota, R.; Kudoh, J.; Mashima, Y.; Asakawa, S.; Minoshima, S.; Hejtmancik, J.F.; Oguchi, Y.; Shimizu, N. Genomic organization of the human myocilin gene (MYOC) responsible for primary open angle glaucoma (GLC1A). Biochem. Biophys. Res. Commun. 1998, 242, 396-400.

41. Mukhopadhyay, A.; Talukdar, S.; Bhattacharjee, A.; Ray, K. Bioinformatic approaches for identification and characterization of olfactomedin related genes with a potential role in pathogenesis of ocular disorders. Mol. Vis. 2004, 10, 304-314.

42. Yokoe, H.; Anholt, R.R. Molecular cloning of olfactomedin, an extracellular matrix protein specific to olfactory neuroepithelium. Proc. Natl. Acad. Sci. USA 1993, 90, 4655-4659.

43. Zeng, L.C.; Han, Z.G.; Ma, W.J. Elucidation of subfamily segregation and intramolecular coevolution of the olfactomedin-like proteins by comprehensive phylogenetic analysis and gene expression pattern assessment. FEBS Lett. 2005, 579, 5443-5453.

44. Snyder, D.A.; Rivers, A.M.; Yokoe, H.; Menco, B.P.; Anholt, R.R. Olfactomedin: Purification, characterization, and localization of a novel olfactory glycoprotein. Biochemistry 1991, 30, 9143-9153.

45. Barembaum, M.; Moreno, T.A.; LaBonne, C.; Sechrist J.; Bronner-Fraser, M. Noelin-1 is a secreted glycoprotein involved in generation of the neural crest. Nat. Cell Biol. 2000, 2, 219-225. 
46. Danielson, P.E.; Forss-Petter, S.; Battenberg, E.L.; deLecea, L.; Bloom, F.E.; Sutcliffe, J.G. Four structurally distinct neuron-specific olfactomedin-related glycoproteins produced by differential promoter utilization and alternative mRNA splicing from a single gene. J. Neurosci. Res. 1994, 38, 468-478.

47. Graveel, C.R.; Harkins-Perry, S.R.; Acevedo, L.G.; Farnham, P.J. Identification and characterization of CRG-L2, a new marker for liver tumor development. Oncogene 2003, 22, 1730-1736.

48. Hillier, B.J.; Vacquier, V.D. Amassin, an olfactomedin protein, mediates the massive intercellular adhesion of sea urchin coelomocytes. J. Cell Biol. 2003, 160, 597-604.

49. Kulkarni, N.H.; Karavanich, C.A.; Atchley, W.R.; Anholt, R.R. Characterization and differential expression of a human gene family of olfactomedin-related proteins. Genet. Res. 2000, 76, 41-50.

50. Loria, P.M.; Hodgkin, J.; Hobert, O. A conserved postsynaptic transmembrane protein affecting neuromuscular signaling in Caenorhabditis elegans. J. Neurosci. 2004, 24, 2191-2201.

51. Nakaya, N.; Lee, H.S.; Takada, Y.; Tzchori, I.; Tomarev, S.I. Zebrafish olfactomedin 1 regulates retinal axon elongation in vivo and is a modulator of Wnt signaling pathway. J. Neurosci. 2008, 28, 7900-7910.

52. Torrado, M.; Trivedi, R.; Zinovieva, R.; Karavanova, I.; Tomarev, S.I. Optimedin: A novel olfactomedin-related protein that interacts with myocilin. Hum. Mol. Genet. 2002, 11, 1291-1301.

53. Tsuda, H.; Sasai, N.; Matsuo-Takasaki, M.; Sakuragi, M.; Murakami, Y.; Sasai, Y. Dorsalization of the neural tube by Xenopus tiarin, a novel patterning factor secreted by the flanking nonneural head ectoderm. Neuron 2002, 33, 515-528.

54. Aroca-Aguilar, J.D.; Sanchez-Sanchez, F.; Ghosh, S.; Coca-Prados, M.; Escribano, J. Myocilin mutations causing glaucoma inhibit the intracellular endoproteolytic cleavage of myocilin between amino acids arg226 and ile227. J. Biol. Chem. 2005, 280, 21043-21051.

55. Tamm, E.R. Myocilin and glaucoma: Facts and ideas. Prog. Retin. Eye Res. 2002, 21, 395-428.

56. Ortego, J.; Escribano, J.; Coca-Prados, M. Cloning and characterization of subtracted cDNAs from a human ciliary body library encoding TIGR, a protein involved in juvenile open angle glaucoma with homology to myosin and olfactomedin. FEBS Lett. 1997, 413, 349-353.

57. Gobeil, S.; Letartre, L.; Raymond, V. Functional analysis of the glaucoma-causing TIGR/myocilin protein: integrity of amino-terminal coiled-coil regions and olfactomedin homology domain is essential for extracellular adhesion and secretion. Exp. Eye Res. 2006, 82, 1017-1029.

58. Burns, J.N.; Orwig, S.D.; Harris, J.L.; Watkins, J.D.; Vollrath, D.; Lieberman, R.L. Rescue of glaucoma-causing mutant myocilin thermal stability by chemical chaperones. ACS Chem. Biol. 2010, 5, 477-487.

59. Fingert, J.H.; Stone, E.M.; Sheffield, V.C.; Alward, W.L. Myocilin glaucoma. Surv. Ophthalmol. 2002, 47, 547-561.

60. Kim, B.S.; Savinova, O.V.; Reedy, M.V.; Martin, J.; Lun, Y.; Gan, L.; Smith, R.S.; Tomarev, S.I.; John, S.W.M.; Johnson, R.L. Targeted disruption of the myocilin gene (Myoc) suggests that human glaucoma-causing mutations are gain of function. Mol. Cell. Biol. 2001, 21, 7707-7713.

61. Gould, D.B.; Miceli-Libby, L.; Savinova, O.V.; Torrado, M.; Tomarev, S.I.; Smith, R.S.; John, S.W.M. Genetically increasing Myoc expression supports a necessary pathologic role of abnormal proteins in glaucoma. Mol. Cell. Biol. 2004, 24, 9019-9025. 
62. Wiggs, J.L.; Vollrath, D. Molecular and clinical evaluation of a patient hemizygous for TIGR/MYOC. Arch. Ophthal. 2001, 119, 1674-1678.

63. Lam, D.S.; Leung, Y.F.; Chua, J.K.; Baum, L.; Fan, D.S.; Choy, K.W.; Pang, C.P. Truncations in the TIGR gene in individuals with and without primary open-angle glaucoma. Investig. Ophthalmol. Vis. Sci. 2000, 4, 1386-1391.

64. Paper, W.; Kroeber, M.; Heersink, S.; Stephan, D.A.; Fuchshofer, R.; Russell, P.; Tamm, E.R. Elevated amounts of myocilin in the aqueous humor of transgenic mice cause significant changes in ocular gene expression. Exp. Eye Res. 2008; 87, 257-267.

65. Fautsch, M.P.; Vrabel, A.M.; Johnson, D.H. The identification of myocilin-associated proteins in the human trabecular meshwork. Exp. Eye Res. 2006, 82, 1046-1052.

66. Joe, M.K.; Sohn, S.; Choi, Y.R.; Park, H.; Kee, C. Identification of flotillin-1 as a protein interacting with myocilin: Implications for the pathogenesis of primary open-angle glaucoma. Biochem. Biophys. Res. Commun. 2005, 336, 1201-1206.

67. Li, Y.; Aroca-Aguilar, J.D.; Ghosh, S.; Sánchez-Sánchez, F.; Escribano, J.; Coca-Prados, M. Interaction of myocilin with the C-terminal region of hevin. Biochem. Biophys. Res. Commun. 2006, 339, 797-804.

68. Peters, D.M.; Herbert, K.; Biddick, B.; Peterson, J.A. Myocilin binding to Hep II domain of fibronectin inhibits cell spreading and incorporation of paxillin into focal adhesions. Exp. Cell Res. 2005, 303, 218-228.

69. Surgucheva, I.; Park, B.C.; Yue, B.Y.; Tomarev, S.; Surguchov, A. Interaction of myocilin with gamma-synuclein affects its secretion and aggregation. Cell. Mol. Neurobiol. 2005, 25, 1009-1033.

70. Kwon, H.S.; Lee, H.S.; Ji, Y.; Rubin, J.S.; Tomarev, S.I. Myocilin is a modulator of Wnt signaling. Mol. Cell. Biol. 2009, 29, 2139-2154.

71. Wentz-Hunter, K.; Ueda, J.; Yue, B.Y. Protein interactions with myocilin. Invest. Ophthalmol. Vis. Sci. 2002, 43, 176-182.

72. Copin, B.; Brézin, A.P.; Valtot, F.; Dascotte, J.C.; Béchetoille, A.; Garchon, H.J. Apolipoprotein E-promoter single-nucleotide polymorphisms affect the phenotype of primary open-angle glaucoma and demonstrate interaction with the myocilin gene. Am. J. Hum. Genet. 2002, 70, 1575-1581.

73. Ueda, J.; Yue, B.Y. Distribution of myocilin and extracellular matrix components in the corneoscleral meshwork of human eyes. Invest. Ophthalmol. Vis. Sci. 2003, 44, 4772-4779.

74. Fautsch, M.P.; Johnson, D.H. Characterization of myocilin-myocilin interactions. Invest. Ophthalmol. Vis. Sci. 2001, 42, 2324-2331.

75. Lo, W.R.; Rowlette, L.L.; Caballero, M.; Yang, P.; Hernandez, M.R.; Borras, T. Tissue differential microarray analysis of dexamethasone induction reveals potential mechanisms of steroid glaucoma. Invest. Ophthal. Vis. Sci. 2003, 44, 473-485.

76. Liton, P.B.; Luna, C.; Challa, P.; Epstein, D.L.; Gonzalez, P. Genome-wide expression profile of human trabecular meshwork cultured cells, non glaucomatous and primary open angle glaucoma tissue. Mol. Vis. 2006, 12, 774-790.

77. Tomarev, S.I.; Nakaya, N. Olfactomedin domain-containing proteins: Possible mechanisms of action and functions in normal development and pathology. Mol. Neurobiol. 2009, 40, 122-138. 
78. Kwon, Y.H.; Fingert, J.H.; Kuehn, M.H.; Alward, W.L.M. Primary Open-Angle Glaucoma. N. Engl. J. Med. 2009, 360, 1113-1124.

79. Zhou, Z.; Vollrath, D. A cellular assay distinguishes normal and mutant TIGR/myocilin protein. Hum. Mol. Genet. 1999, 8, 2221-2228.

80. Rangachari, K.; Dhivya, M.; Eswari Pandaranayaka, P.J.; Prasanthi, N.; Sundaresan, P.; Krishnadas, S.R.; Krishnaswamy, S. Glaucoma database. Bioinformation 2011, 5, 398-399.

81. Adam, M.F.; Belmouden, A.; Binisti, P.; Brezin, A.P.; Valtot, F.; Bechetoille, A.; Dascot, J.-C.; Copin, B.; Gomez, L.; Chaventre, A.; et al. Recurrent mutations in a single exon encoding the evolutionarily conserved olfactomedin-homology domain of TIGR in familial open-angle glaucoma. Hum. Molec. Genet. 1997, 6, 2091-2097.

82. Colomb, E.; Nguyen, T.D.; Bechetoille, A.; Dascotte, J.-C.; Valtot, F.; Brezin, A.P.; Berkani, M.; Copin, B.; Gomez, L.; Polansky, J.R.; et al. Association of a single nucleotide polymorphism in the TIGR/MYOCILIN gene promoter with the severity of primary open-angle glaucoma. Clin. Genet. 2001, 60, 220-225.

83. Polansky, J.R.; Juster, R.P.; Spaeth, G.L. Association of the myocilin mt.1 promoter variant with the worsening of glaucomatous disease over time. Clin. Genet. 2003, 64, 18-27.

84. Saura, M.; Cabana, M.; Ayuso, C.; Valverde, D. Mutations including the promoter region of myocilin/TIGR gene. Eur. J. Hum. Genet. 2005, 13, 384-387.

85. Kasahara, N.; Caixeta-Umbelino, C.; Paolera, M.D.; Rocha, M.N.; Richeti, F.; Vasconcellos, J.P.; Cohen, R.; Costa, V.P.; Longui, C.A.; Melo, M.R.; et al. Myocilin mt.1 gene promoter single nucleotide polymorphism $(-1000 \mathrm{C}>\mathrm{G})$ in Brazilian patients with primary open angle glaucoma. Ophthal. Genet. 2011, 32, 18-23.

86. Faucher, M.; Anctil, J.-L.; Rodrigue, M.-A.; Duchesne, A.; Bergeron, D.; Blondeau, P.; Cote, G.; Dubois, S.; Bergeron, J.; Arseneault, R.; et al. Founder TIGR/myocilin mutations for glaucoma in the Quebec population. Hum. Mol. Genet. 2002, 11, 2077-2090.

87. Fingert, J.H.; Heon, E.; Liebmann, J.M.; Yamamoto, T.; Craig, J.E.; Rait, J.; Kawase, K.; Hoh, S.-T.; Buys, Y.M.; Dickinson, J.; et al. Analysis of myocilin mutations in 1703 glaucoma patients from five different populations. Hum. Mol. Genet. 1999, 8, 899-905.

88. De Vasconcellos, J.P.; de Melo, M.B.; Schimiti, R.; Costa, F.F.; Costa, V.P. Penetrance and phenotype of the Cys433Arg myocilin mutation in a family pedigree with primary open-angle glaucoma. J. Glaucoma 2003, 12, 104-107.

89. Vincent, A.L.; Billingsley, G.; Buys, Y.; Levin, A.V.; Priston, M.; Trope, G.; Williams-Lyn, D.; Heon, E. Digenic inheritance of early-onset glaucoma: CYP1B1, a potential modifier gene. Am. J. Hum. Genet. 2002, 70, 448-460.

90. Yam, G.H.-F.; Zuber, C.; Roth, J. A synthetic chaperone corrects the trafficking defect and disease phenotype in a protein misfolding disorder. FASEB J. 2005, 19, 12-18.

91. Yam, G.H.; Gaplovska-Kysela, K.; Zuber, C.; Roth, J. Aggregated myocilin induces russell bodies and causes apoptosis: implications for the pathogenesis of myocilin-caused primary open-angle glaucoma. Am. J. Pathol. 2007, 170, 100-109.

92. Aroca-Aguilar, J.D.; Sánchez-Sánchez, F.; Ghosh, S.; Fernández-Navarro, A.; Coca-Prados, M.; Escribano, J. Interaction of Recombinant Myocilin with the Matricellular Protein SPARC: Functional Implications. Invest. Ophthalmol. Vis. Sci. 2011, 52, 179-189. 
93. Sánchez-Sánchez, F.; Martínez-Redondo, F.; Aroca-Aguilar, J.D.; Coca-Prados, M.; Escribano, J. Characterization of the intracellular proteolytic cleavage of myocilin and identification of calpain II as a myocilin-processing protease. J. Biol. Chem. 2007, 282, 27810-27824.

94. Aroca-Aguilar, J.D.; Martínez-Redondo, F.; Sánchez-Sánchez, F.; Coca-Prados, M.; Escribano, J. Functional role of proteolytic processing of recombinant myocilin in self-aggregation. Invest. Ophthalmol. Vis. Sci. 2010, 51, 72-78.

95. Jia, L.-Y.; Gong, B.; Pang, C.-P.; Huang, Y.; Lam, D.S.-C.; Wang, N.; Yam, G.H.-F. Correction of the disease phenotype of myocilin-causing glaucoma by a natural osmolyte. Invest. Ophthalmol. Vis. Sci. 2009, 50, 3743-3749.

96. Yam, G.H.-F.; Gaplovska-Kysela, K.; Zuber, C.; Roth, J. Sodium 4-phenylbutyrate acts as a chemical chaperone on misfolded myocilin to rescue cells from endoplasmic reticulum stress and apoptosis. Invest. Ophthalmol. Vis. Sci. 2007, 48, 1683-1690.

97. Menaa, B.; Menaa, F.; Aiolfi-Guimarães, C.; Sharts, O. Silica-based nanoporous sol-gel glasses: From bioencapsulation to protein folding studies. Int. J. Nanotechnol. 2010, 7, 1-45.

98. Menaa, B.; Montoneri, C.; Menaa, F.; Montoneri, E.; Boffa, V.; Sharts, N. Protein helical structure enhancement in biocompatible fluoro-phosphonate-based nanoporous silica glasses assessed by circular dichroism spectroscopy. Int. J. Nanotechnol. 2011, 8, 471-491.

99. Menaa, B.; Miyagawa, Y.; Takahashi, M.; Herrero, M.; Rives, V.; Menaa, F.; Eggers. D.K. Bioencapsulation of apomyoglobin in nanoporous organosilica sol-gel glasses: Influence of the siloxane network on the conformation and stability of a model protein. Biopolymers 2009, 91, 895-906.

100. Orwig, S.D.; Lieberman, R.L. Biophysical characterization of the olfactomedin domain of myocilin, an extracellular matrix protein implicated in inherited forms of glaucoma. PLoS One 2011, 6, e16347.

101. Thompson, J.D.; Higgins, D.G.; Gibson, T.J. CLUSTAL W: Improving the sensitivity of progressive multiple sequence alignment through sequence weighting, position-specific gap penalties and weight matrix choice. Nucl. Acid. Res. 1994, 22, 4673-4680.

102. Higgins, D.G.; Thompson, J.D.; Gibson, T.J. Using CLUSTAL for multiple sequence alignments. Methods Enzymol. 1996, 266, 383-402.

103. Roy, A.; Kucukural, A.; Zhang, Y. I-TASSER: A unified platform for automated protein structure and function prediction. Nat. Protoc. 2010, 5, 725-738.

104. Zhang, Y. I-TASSER: Fully automated protein structure prediction in CASP8. Proteins 2009, S9, 100-113.

105. Zhang, Y. I-TASSER server for protein 3D structure prediction. BMC Bioinform. 2008, 9, 40.

106. Bluhm, W.F.; Beran, B.; Bi, C.; Dimitropoulos, D.; Prlic, A.; Quinn, G.B.; Rose, P.W.; Shah, C.; Young, J.; Yukich, B.; et al. Quality assurance for the query and distribution systems of the RCSB Protein Data Bank. Database (Oxford) 2011, 2011, Article ID bar003.

107. Rose, P.W.; Beran, B.; Bi, C.; Bluhm, W.F.; Dimitropoulos, D.; Goodsell, D.S.; Prlic, A.; Quesada, M.; Quinn, G.B.; Westbrook, J.D.; et al. The RCSB Protein Data Bank: redesigned web site and web services. Nucl. Acid. Res. 2011, Database, D392-D401. 
108. Suzuki, Y.; Noma, A.; Suzuki, T.; Ishitani, R.; Nureki, O. Structural basis of tRNA modification with $\mathrm{CO} 2$ fixation and methylation by wybutosine synthesizing enzyme TYW4. Nucl. Acid. Res. 2009, 37, 2910-2925.

Sample Availability: Samples of the compounds are available from the authors.

(C) 2011 by the authors; licensee MDPI, Basel, Switzerland. This article is an open access article distributed under the terms and conditions of the Creative Commons Attribution license (http://creativecommons.org/licenses/by/3.0/). 